\title{
Impact of Domestic Violence on Postpartum Depression among Women in Rey City, Iran, in 2016
}

\author{
Parisa Akhbary $^{1}$, Hossein Mohsenipouya ${ }^{2}$, Davood Shojaeizadeh ${ }^{3, *}$, Abolhassan Naghibi ${ }^{4}$ \\ ${ }^{I}$ MSc in Health Education and Promotion, Shahid Beheshti University of Medical Sciences, Tehran, Iran \\ ${ }^{2}$ PhD in Health Education and Promotion, Cardiovascular Research Center, Mazandaran University of Medical Sciences, \\ Sari, Iran \\ ${ }^{3}$ Professor, Department of Health Education and Promotion, School of Public Health, Tehran University of Medical Sciences, \\ Tehran, Iran \\ ${ }^{4}$ Assistant Professor of Public Health, Health Sciences Research Center, Mazandaran University of Medical Sciences, Sari, \\ Iran
}

* Corresponding Author: Davood Shojaeizadeh, Department of Health Education and Promotion, School of Public Health, Tehran University of Medical Sciences, Tehran, Iran. Email: shojaei@tums.ac.ir

\begin{abstract}
Received: 03.09.2017
Accepted: 15.01 .2018

How to Cite this Article: Akhbary P, Mohsenipouya $\mathrm{H}$, Shojaeizadeh D, Naghibi A. Impact of Domestic Violence on Postpartum Depression among Women in Rey City, Iran, in 2016. Avicenna J Clin Med. 2018; 24(4): 299-306. DOI: 10.21859 / ajcm.24.4.299.

\section{Abstract}

Background and Objective: Domestic violence during pregnancy poses the mothers to physical and mental health problems in the future. The aim of this study was to evaluate the effect of domestic violence on postpartum depression among the women referring to the healthcare centers in Rey city, Iran.

Materials and Methods: This descriptive-analytical, cross-sectional study was conducted on 120 females referring to the healthcare centers of Rey city in 2016. The study population was selected through convenience sampling technique. The data were collected using a questionnaire including three parts, including demographic information (10 items), Edinburgh Postnatal Depression Scale, and standard questionnaire of domestic violence. Data analysis was performed in SPSS software (version 22) using t-test and Pearson correlation coefficient.

Results: There was a significant positive association between physical violence and postpartum depression $(\mathrm{P}<0.05)$. However, postpartum depression showed no significant association with economic and psychological violence $(\mathrm{P}>0.05)$.

Conclusion: As the findings indicated, physical violence could lead to the enhancement of postpartum depression among the women. Therefore, it is essential to take some measures targeted toward the reduction of physical violence by using appropriate educational and interventional approaches and encouraging the healthcare workers to establish an intimate relationship with the pregnant women.
\end{abstract}

Keywords: Domestic Violence, Postpartum Depression, Women 
d०): $10.21859 /$ ajcm.24.4.299

\title{
بررسى تأثير خشونت خانكى بر افسردتى يس از زايمان زنان ساكن شهر رى در سال
}

\section{1 rad}

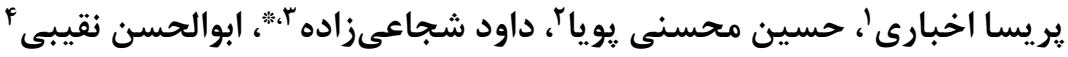 \\ ' كارشناسى ارشد آموزش بهداشت و ارتقاى سلامت، دانشعاه علوم يزشكى شهيد بهشتى، تهران، ايران

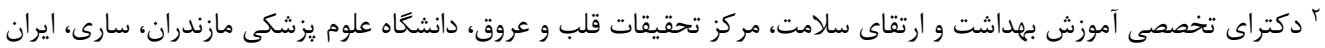

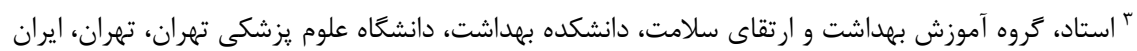

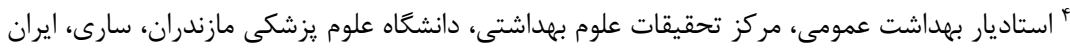
* نويسنده مسئول: داود شجاعىزاده، گروه آموزش بهداشت و ارتقاى سلامت، دانشكده بهداشت، دانشكاه علوم يزشكى تهران، تهران، ايران. ايميل: shojaei@tums.ac.ir

\begin{tabular}{|c|c|}
\hline 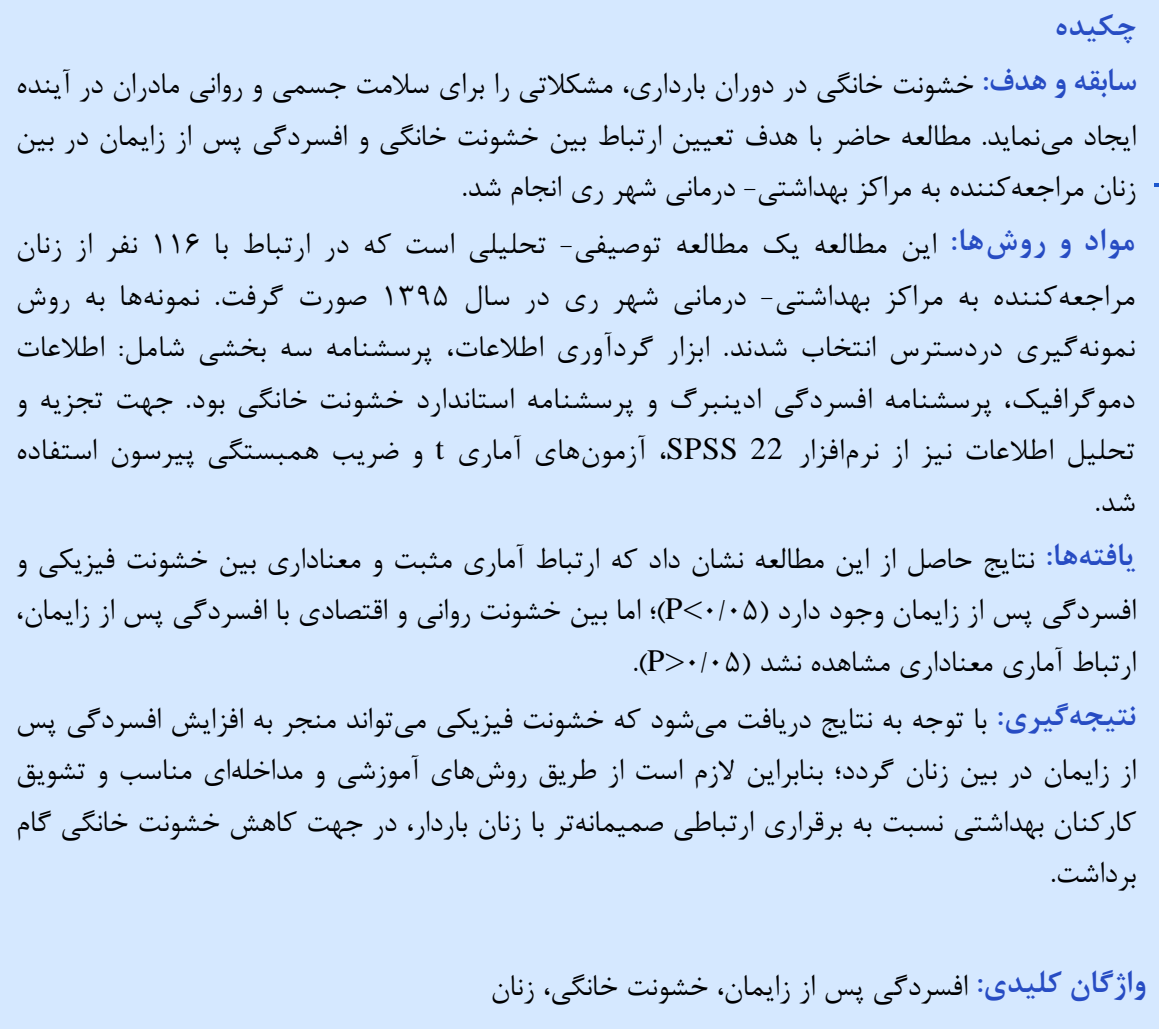 & 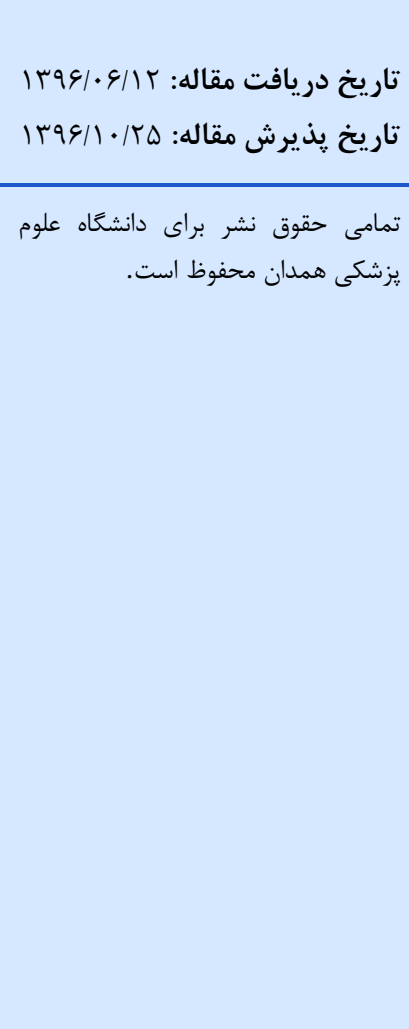 \\
\hline
\end{tabular}

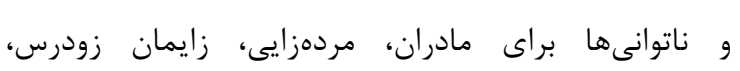

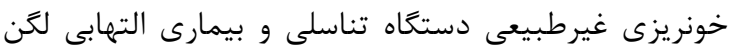

شود [ب].

شيوع خشونت خانكى اعمالشده در باردارى حدود

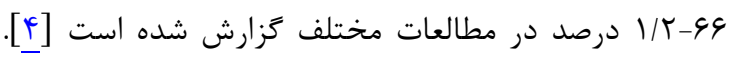

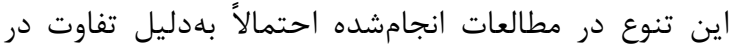

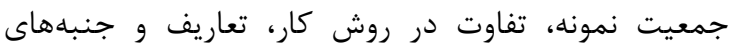

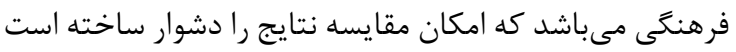

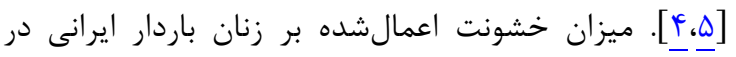

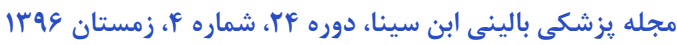

خشونت خانكى در دوران باردارى يكى از موضوعات

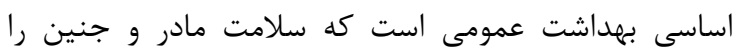

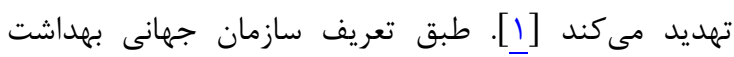
خانك) WhHO: World Health Organization) خانكى در دوران باردارى بهعنوان خشونت جسمى، جنسى يا

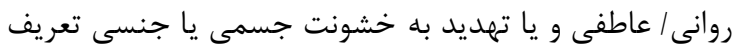

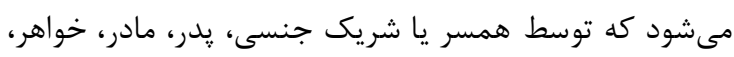

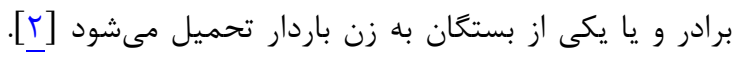

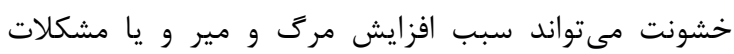


كه اين علائم داراى ارتباطى معنادار با تجربه خشونت دوران

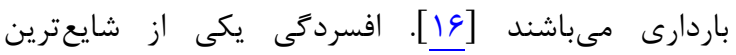
بيمارىهاى روانى در ميان جوامع مختلف است كه دارئ در سال

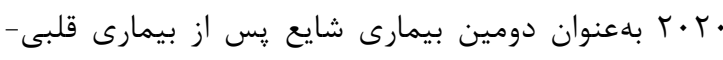

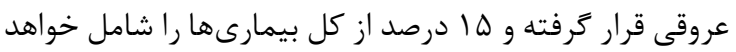

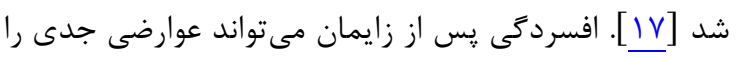

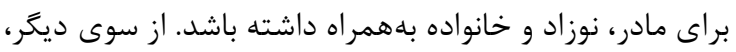

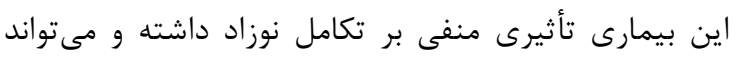

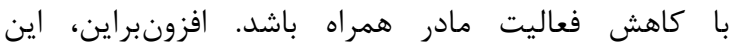

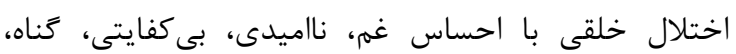

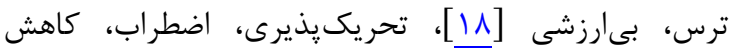

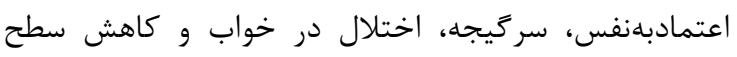
مراقبت از نوزاد همراه است [9 19]؛ ازاينرو، ادامه اين اختلال

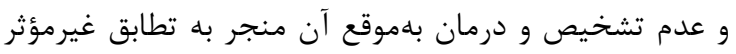

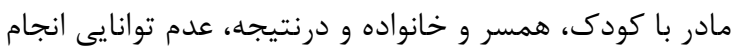

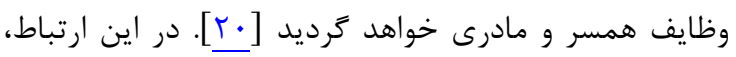

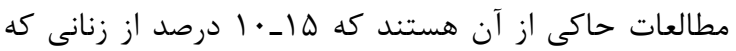

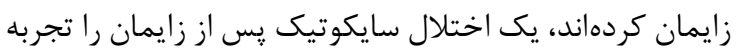

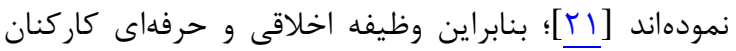

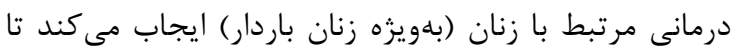

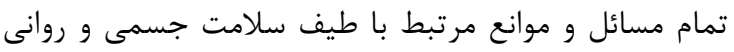

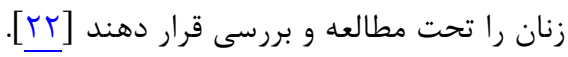

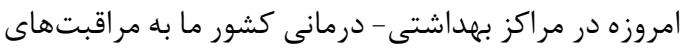

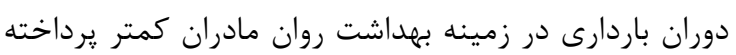

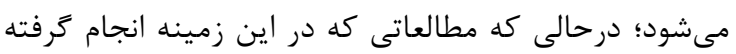

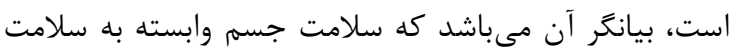

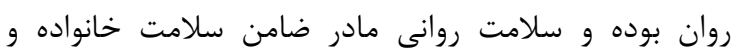

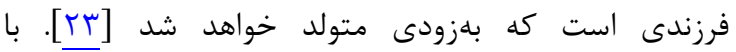

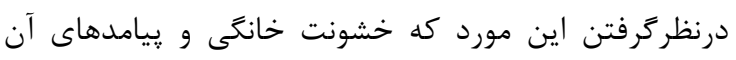

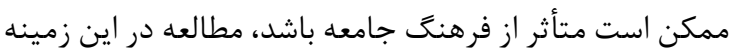

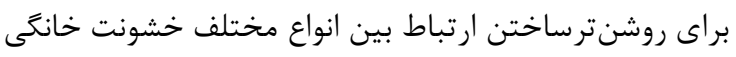

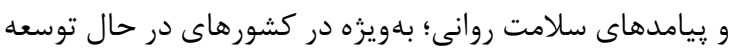

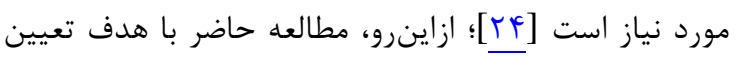

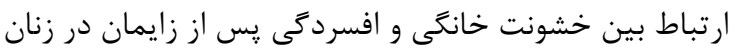
ساكن شهر رى صورت كرفت.

\section{مواد و روشها}

مطالعه توصيفى- تحليلى حاضر با هدف تعيين تأثير

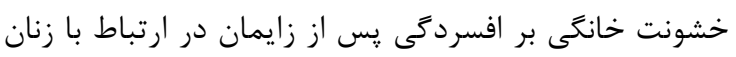

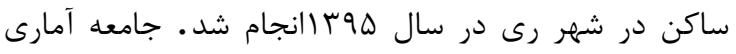

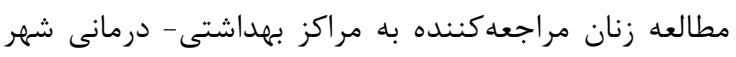

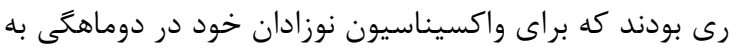

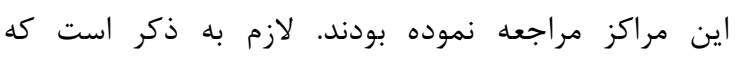

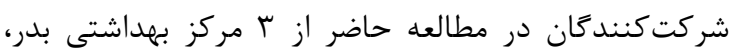

مطالعات انجامشده بيش از •9 درصد كزارش شده است [ع]؛؛

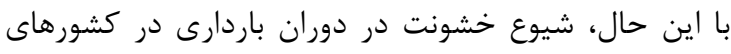

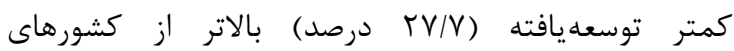

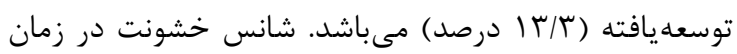

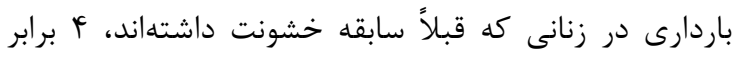

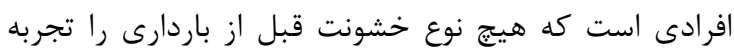

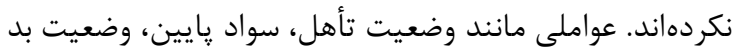

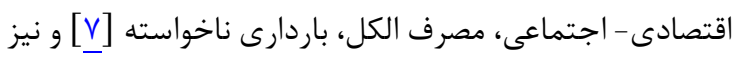

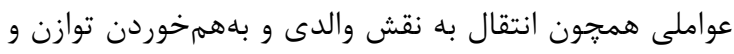

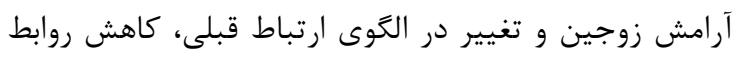

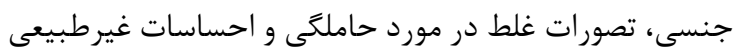

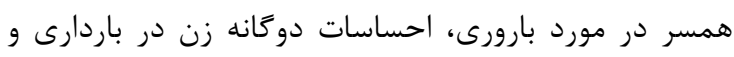

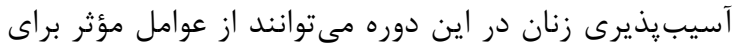

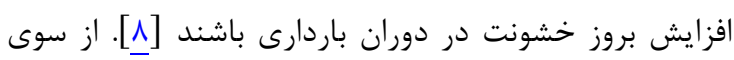

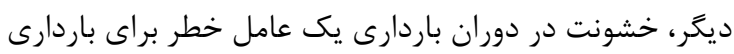

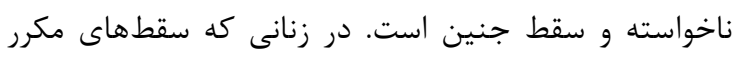

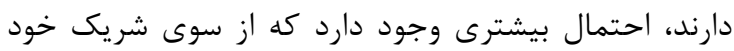

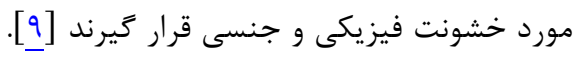

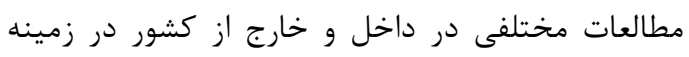

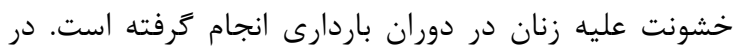

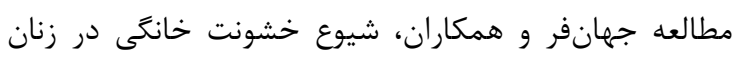
حامله مراجعه كننده به بيمار ستان هاى وابسته

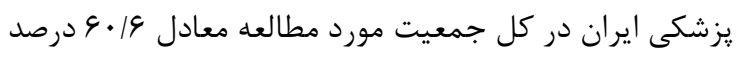

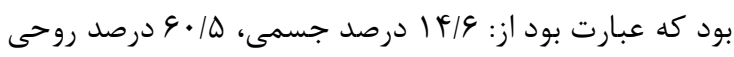

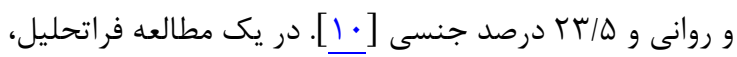

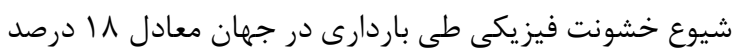

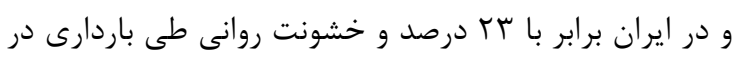

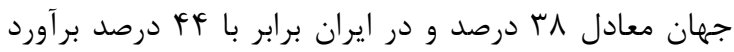

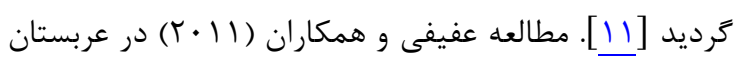

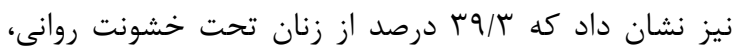

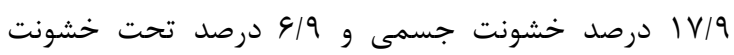

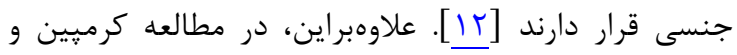

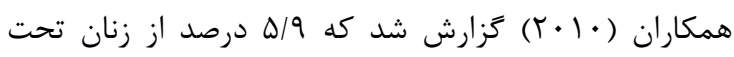

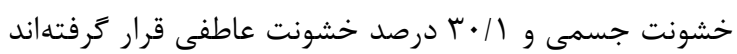

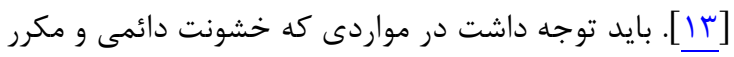

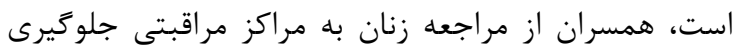

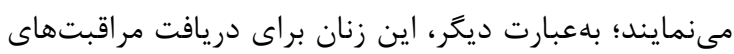

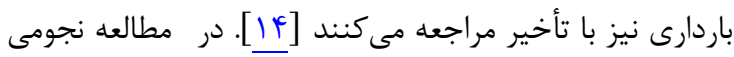

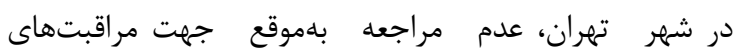

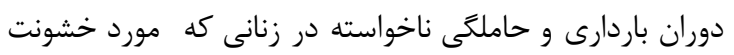

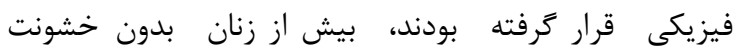
بود [10] سطوح بالايى از اختلالات روانى مانند افسردَى، اضطراب

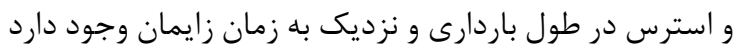


خشونت اقتصادى مىباشند. با توجه به يرسشنامه، هر سؤال

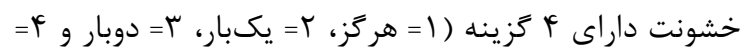

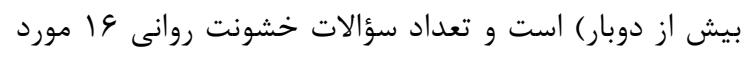

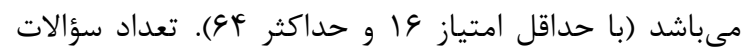

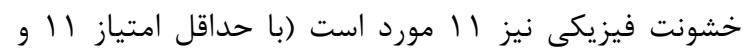

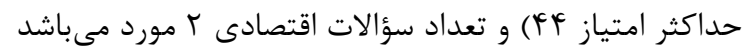

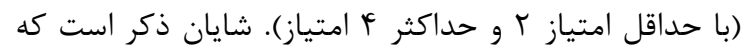

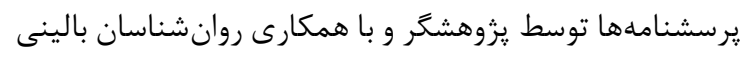
مراكز بهداشتى بهصورت مصاحبه عميق بالينى تكميل ترديد.

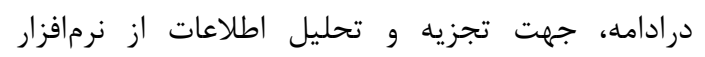
SPSS 22

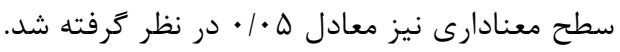

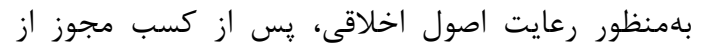

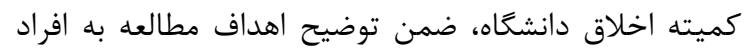

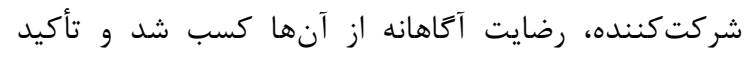

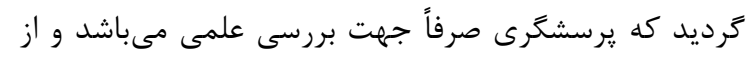

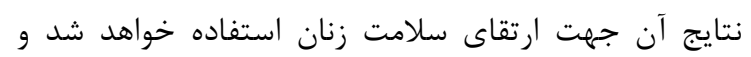
اطلاعات بهصورت كاملاً محرمانه باقى خواهد ماند.

\section{بافته ها}

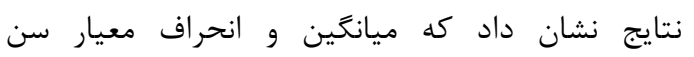

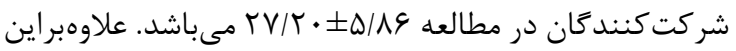

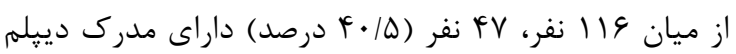
و 1· أفر (NV/I درصد) خانهدار بودند، ميزان تحصيلات

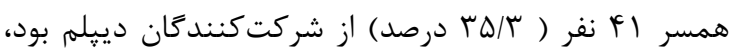

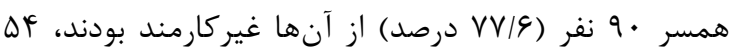
نفر (\&/\& درصد) از زنان مورد مطالعه سابقه سقط نداشتند و و •9 نفر

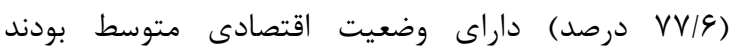

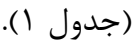

ميانكين و انحراف معيار انواع خشونت عليه زنان در جدول

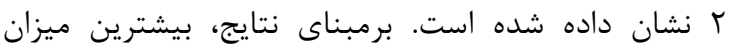

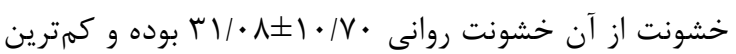

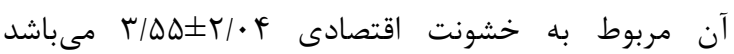

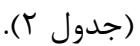

بهمنظور بررسى رابطه بين خشونت روانى، فيزيكى و

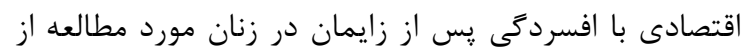

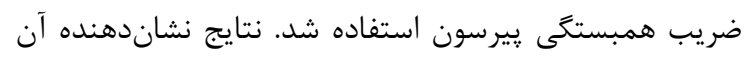

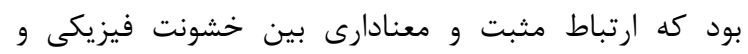

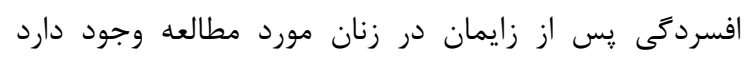

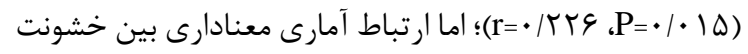

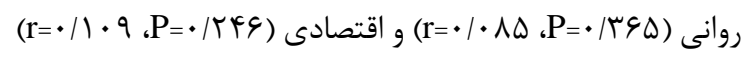

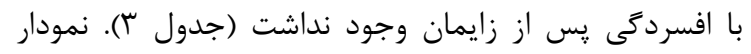

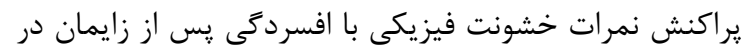

شهيد جمالى و شور آباد به شيوه نمونه انتخاب شدند. معيارهاى ورود به مطالعه عبارت بودند از: قرارداشتن در

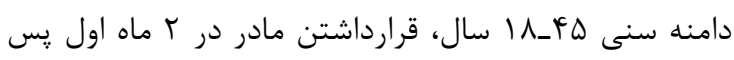

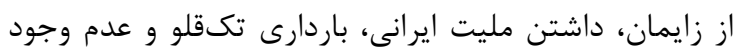

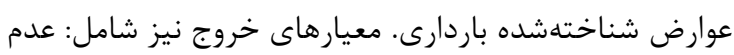
رضايت براى شركت در مطالعه، وجود بيمارىهاى روحيى و

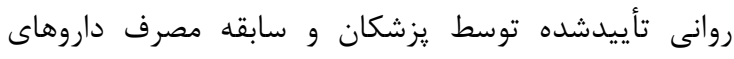

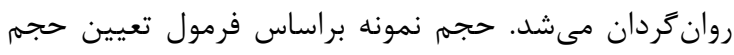

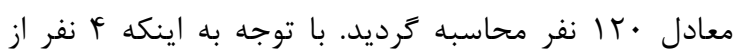
شركت كنند

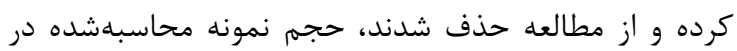

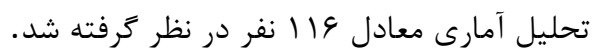

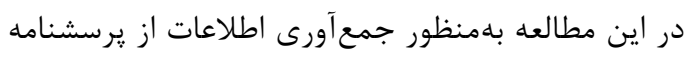

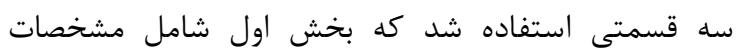

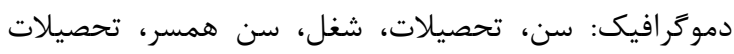

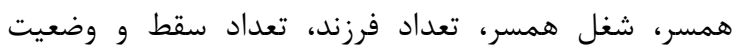
اقتصادى بود و بخش دوم مربوط به همبه يرسشنامه استاندارد ادينبرى (Edinburgh Postnatal Depression Scale)

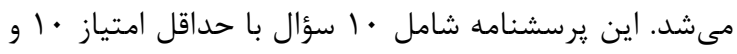

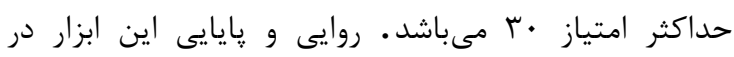

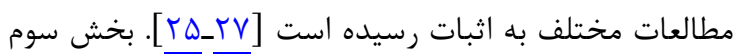

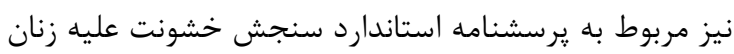

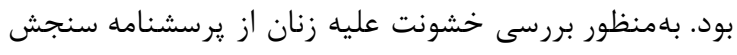

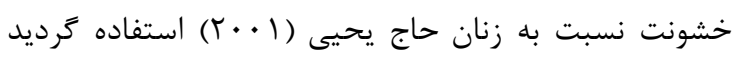

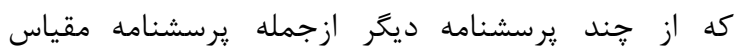

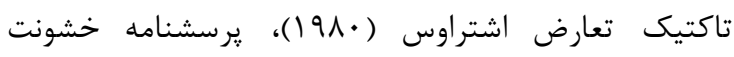

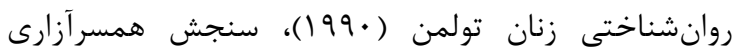

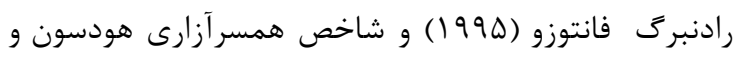

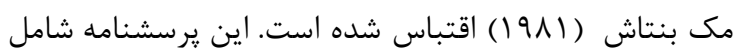

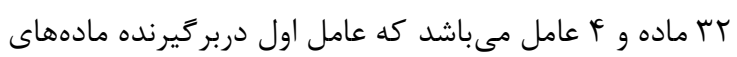

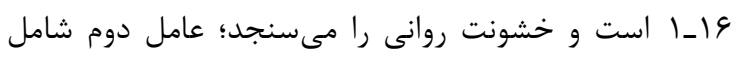

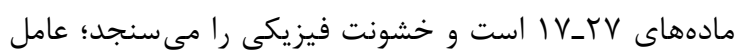

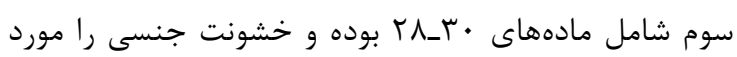

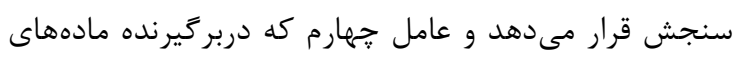

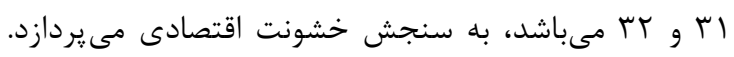

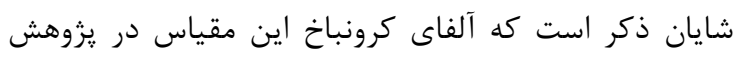

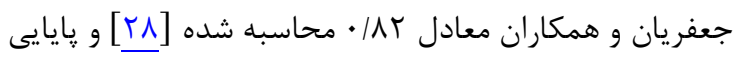
كل مقياس در يزوهش لطيفيان و همكاران با استفاده از آلفاى همان

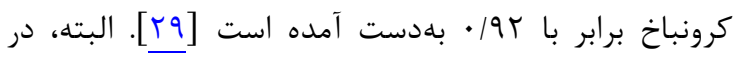

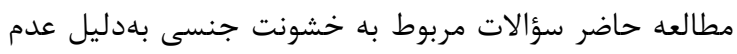

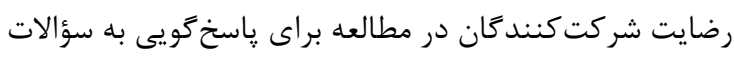

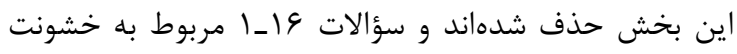

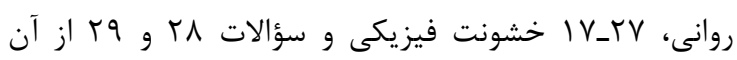


جدول ا: مشخصات جمعيتى زنان تحت مطالعه

\begin{tabular}{|c|c|c|}
\hline درصد & فراوانى & \\
\hline & & تحصيلات \\
\hline$r F / l$ & $r \Lambda$ & بىسواد \\
\hline$r \& / V$ & r & زيردييلم \\
\hline$r \cdot 10$ & iv & دييلم \\
\hline \multirow[t]{2}{*}{$1 / 9$} & 1. & دانشخاهى \\
\hline & & شغل \\
\hline$\Lambda \vee / 1$ & $1 \cdot 1$ & 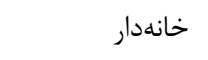 \\
\hline $1 / 9$ & 1. & كارمند \\
\hline \multirow[t]{2}{*}{$\boldsymbol{k} / \mu$} & $\Delta$ & غير كارمند \\
\hline & & تحصيلات همسر \\
\hline$I V / T$ & $r$. & 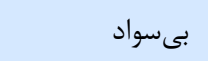 \\
\hline$r \Delta / r$ & (i) & زيردييلم \\
\hline ( ) & rq & دييلم \\
\hline \multirow[t]{2}{*}{ Ir/A } & 19 & دانشخاهى \\
\hline & & شغل همسر \\
\hline$r / 9$ & r & بيكار \\
\hline $19 / 1$ & rז & كارمند \\
\hline \multirow[t]{2}{*}{$V V / S$} & 9. & غير كارمند \\
\hline & & تعداد فرزند \\
\hline$p \& / 9$ & $\Delta F$ & يكى \\
\hline$r \cdot r$ & ra & دو \\
\hline \multirow[t]{2}{*}{$r / T$} & tr & سه و بيشتر \\
\hline & & تعداد سقط \\
\hline $99 / 1$ & $\wedge 1$ & صفر \\
\hline rr/r & TV & يك \\
\hline \multirow[t]{2}{*}{$9 / 9$} & $\wedge$ & دو و بيشتر \\
\hline & & وضعيت اقتصادى \\
\hline$r \cdot / V$ & TF & ضعيف \\
\hline$V V / G$ & $q$. & متوسط \\
\hline $1 / V$ & $r$ & خوب \\
\hline
\end{tabular}

جدول r: ميانگين و انحراف معيار انواع خشونت عليه زنان

\begin{tabular}{|c|c|c|c|}
\hline خشونت اقتصادى & خشونت فيزيكى & خشونت روانى & \\
\hline$r / \Delta \Delta$ & $1 F / 94$ & $M I / . \Lambda$ & ميانغين \\
\hline$T / \cdot F$ & $9 / 0 \Lambda$ & $1 \cdot / V$ & انحراف معيار \\
\hline$r_{-} F$ & 11 FF & $19 \_94$ & محدوده نمرات \\
\hline
\end{tabular}

جدول سّ: بررسى ارتباط بين خشونت روانى، فيزيكى و اقتصادى با افسردىى پس از زايمان در زنان مورد مطالعه

\begin{tabular}{|c|c|c|}
\hline س از زايمان & & \\
\hline ضر يب همبستخَى بيرسون (r) & قدار احتمال & \\
\hline$\cdot / \cdot \wedge \Delta$ & 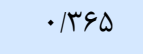 & خشونت روانى \\
\hline 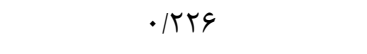 & $.1 \cdot 10$ & خشونت فيزيكى \\
\hline$\cdot 11 \cdot 9$ & - MFE & خشونت اقتصادى \\
\hline
\end{tabular}


خشونت روانى (9V/9 درصد) است. در اين مطالعه انواع

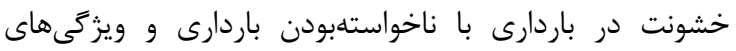

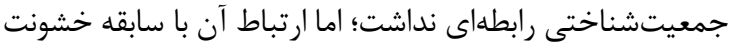

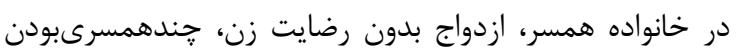

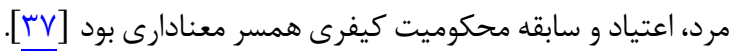

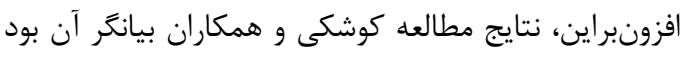

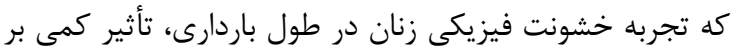

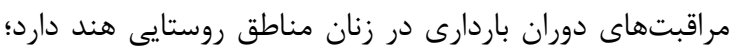

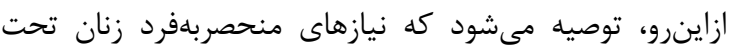

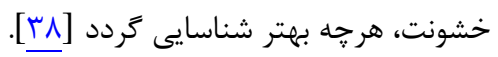

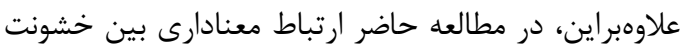

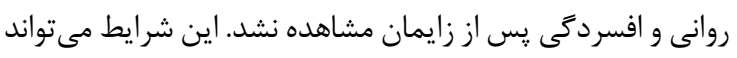

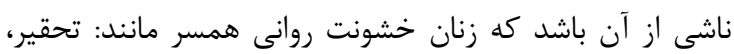

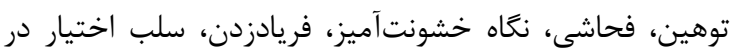
انجامدادن امور شخصى و غيره را ناشى از اعمال نادرست خود

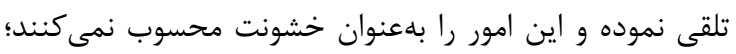

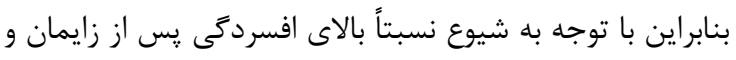

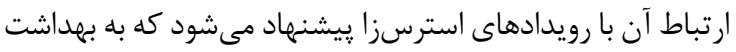

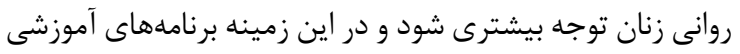

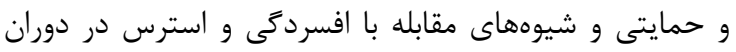

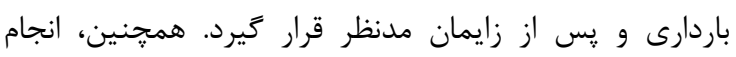

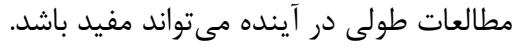
از محدوديتهاى اين مطالعه مىتوان به اين موارد اشاره كرد: 1. بسيارى از شركت كنند

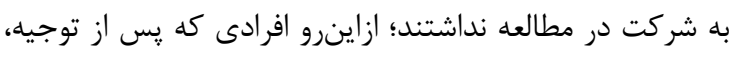

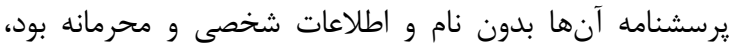

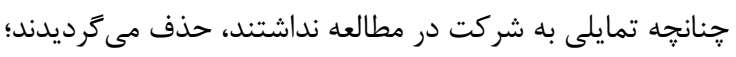

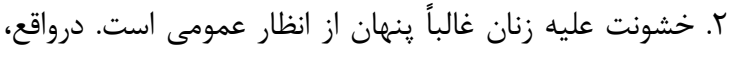

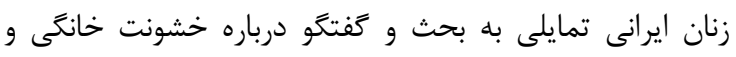

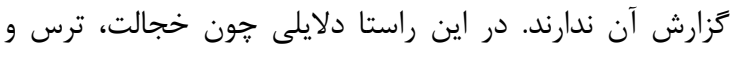

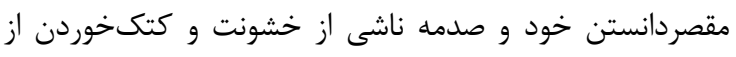
شوهر نيز سبب مىشود كه زنان اين موضوع را مطرح نكنند (اين موضوع نيز مىتوانست باعث سوكيرى در نتايج مطالعه شود).

\section{نتيجه تيرى}

نتايج مطالعه حاضر نشان داد كه يكى از عوارض خشونت در

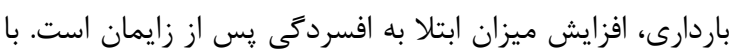

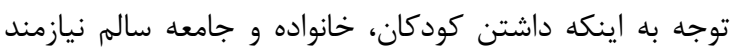

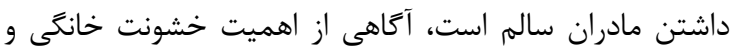
عوارض آن قابل تأمل و راهخشا مىباشد.

\section{تشكر و قدر فاذى}

اين مقاله بركرفته از پايان نامه دانشجويى مقطع كارشناسى

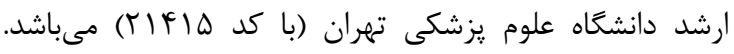

شكل ا نشان داده شده است.

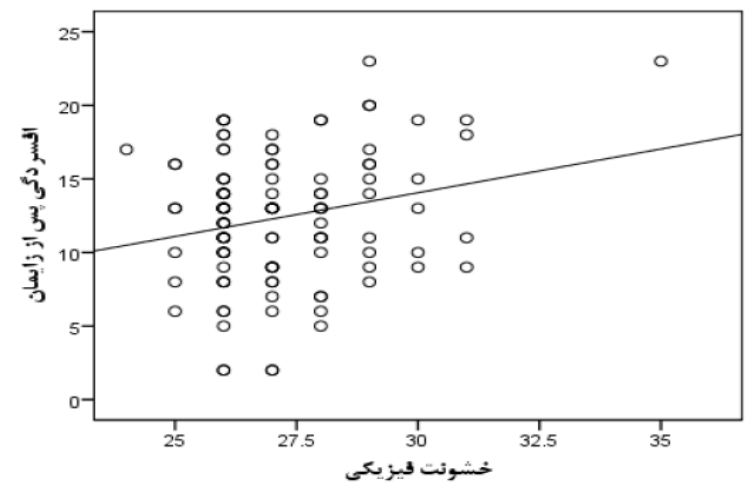

شكل ا: پراكنش خشونت فيزيكى و افسردگى پِ از زايمان در زنان مورد مطالعه

بج نتايج مطالعه حاضر نشان داد كه بين خشونت فيزيكى در

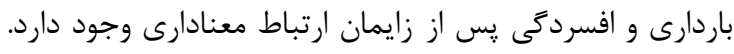

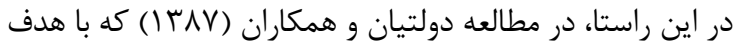

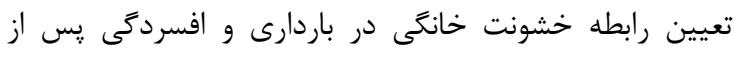

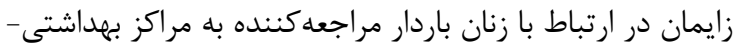

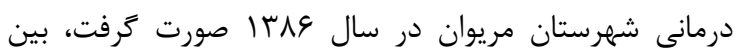

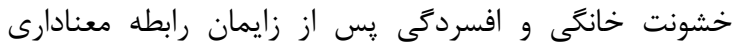

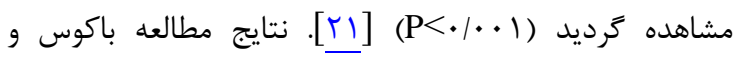

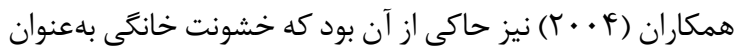

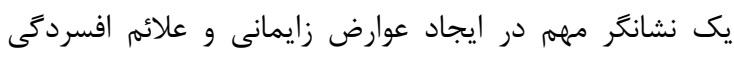
محسوب مىشود. شايان توجه است كه يافتههاى اين مطالعه لزوم آنمانى

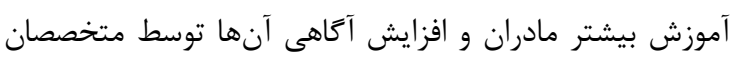

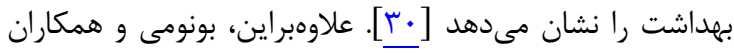

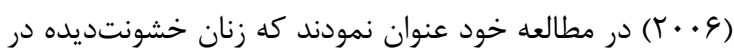

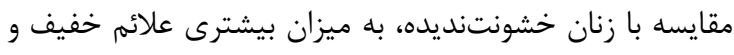

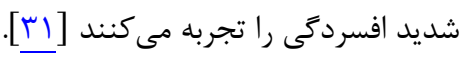
از سوى ديگر، در مطالعه حاضر زنان ميزان خشونت روانى

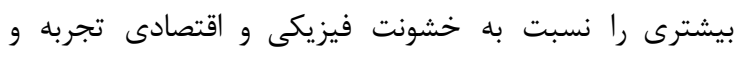

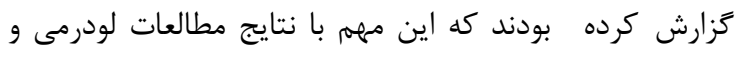

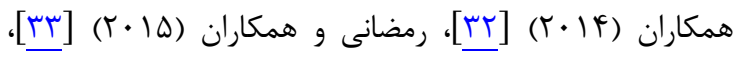

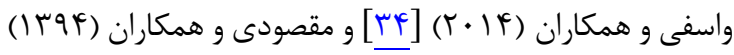

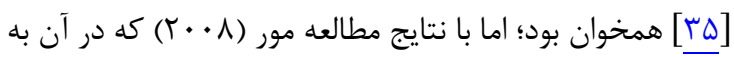

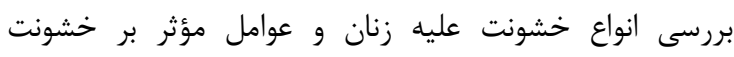

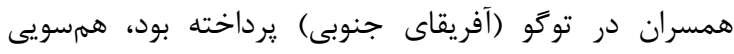

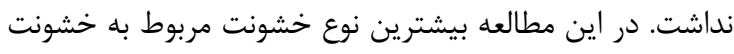

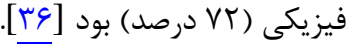

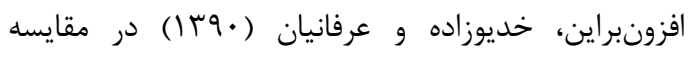
خشونتهاى خانكى، قبل و طى باردارى و عوامل مرتبط با آن

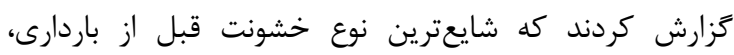


مىدارند. لازم به ذكر است كه هيجَّونه تضاد منافعى در اين مطالعه كزارش نشده است.

\section{REFERENCES}

1. World Health Organization. Violence against women: intimate partner and sexual violence against women: intimate partner and sexual violence have serious short-and long-term physical, mental and sexual and reproductive health problems for survivors. Geneva: World Health Organization; 2014.

2. Centers for Disease Control and Prevention. Intimate partner violence during pregnancy: a guide for clinicians. Georgia: Centers for Disease Control and Prevention; 2006.

3. Ismayilova L. Intimate partner violence in three former Soviet Union countries (Azerbaijan, Moldova, and Ukraine): prevalence, risk factors, and women's reproductive health. New York: Columbia University; 2009.

4. Jasinski JL. Pregnancy and domestic violence: a review of the literature. Trauma Violence Abuse. 2004;5(1):47-64. PMID: 15006296 DOI: $10.1177 / 1524838003259322$

5. Garcia-Moreno C, Heise L, Jansen HA, Ellsberg M, Watts C. Violence against women. Science. 2005;310(5752):1282-3. PMID: 16311321 DOI: $10.1126 /$ science 1121400

6. Hasheminasab L. Prevalence, consequences and factors accompanying with domestic violence in pregnant women referring to sanandaj Labor unit. Sci J Kurdistan Univ Med Sci. 2006;4:32-42.

7. James L, Brody D, Hamilton Z. Risk factors for domestic violence during pregnancy: a meta-analytic review. Violence Vict. 2013;28(3):359-80. PMID: 23862304

8. Hesami K, Dolatian M, Shams J, Majd HA. Domestic violence before and during pregnancy among pregnant women. Iran J Nurs. 2010;23(63):51-9. [Persian]

9. Pallitto CC, García-Moreno C, Jansen HA, Heise L, Ellsberg M, Watts C. Intimate partner violence, abortion, and unintended pregnancy: results from the WHO Multi-country Study on Women's Health and Domestic Violence. Int J Gynecol Obstet. 2013;120(1):3-9. PMID: 22959631 DOI: 10.1016/j.ijgo.2012.07.003

10. Jahanfar S, Malekzadegan Z. The prevalence of domestic violence among pregnant women who were attended in Iran University of Medical Science Hospitals. J Fam Violence. 2007;22(8):643.

11. Karimi A, Daliri S, Sayehmiri K. The prevalence of physical and psychological violence during pregnancy in Iran and the world: a systematic review and meta-analysis. J Clin Nurs Midwifery. 2016;5(3):73-88. [Persian]

12. Afifi Z, Al-Muhaideb NS, Hadish NF, Ismail FI, Al-Qeamy FM. Domestic violence and its impact on married women's health in Eastern Saudi Arabia. Saudi Med J. 2011;32(6):61220. PMID: 21666945

13. Crempien RC, Rojas G, Cumsille P, Oda M. Domestic violence during pregnancy and mental health: exploratory study in primary health centers in Peñalolén. ISRN Obstet Gynecol. 2010;2011:265817. PMID: 21637356 DOI: $10.5402 / 2011 / 265817$

14. Sh J, Malekzadegan A, Jamshidi R. Prevalence of domestic violence in pregnant women referring to the hospitals of Iran University of Medical Sciences. Iran Nurs Quart. 2002;2003:32-3.

15. Hassan M, Kashanian M, Hassan M, Roohi M, Yusefi H. Assessment of association between domestic violence during pregnancy with fetal outcome. Iran J Obstet Gynecol Infertil. 2013;16(43):21-9. [Persian]

16. Howard LM, Oram S, Galley H, Trevillion K, Feder G. Domestic violence and perinatal mental disorders: a systematic review and meta-analysis. PLoS Med. 2013;10(5):e1001452. PMID: 23723741 DOI: 10.1371/ journal.pmed.1001452

17. Beydoun HA, Beydoun MA, Kaufman JS, Lo B, Zonderman $\mathrm{AB}$. Intimate partner violence against adult women and its association with major depressive disorder, depressive symptoms and postpartum depression: a systematic review and meta-analysis. Soc Sci Med. 2012;75(6):959-75. PMID: 22694991 DOI: $10.1016 /$ j.socscimed.2012.04.025

$$
\begin{aligned}
& \text { بدينوسيله نويسندگان مراتب تشكر و قدردانى خود را از مديران، } \\
& \text { كاركنان و مراجعين محترم به مراكز بهداشتى - درمانى شهر رى } \\
& \text { كه صميمانه براى انجام اين مطالعه همكارى نمودند، اعلام }
\end{aligned}
$$

18. Suri R, Stowe ZN, Cohen LS, Newport DJ, Burt VK, AquinoElias AR, et al. Prospective longitudinal study of predictors of postpartum-onset depression in women with a history of major depressive disorder. J Clin Psychiatry. 2017; 78(8):1110-6. PMID: 28297589 DOI: $10.4088 / \mathrm{JCP}$. $15 \mathrm{~m} 10427$

19. Zhao Y, Kane I, Wang J, Shen B, Luo J, Shi S. Combined use of the Postpartum Depression Screening Scale (PDSS) and Edinburgh Postnatal Depression Scale (EPDS) to identify antenatal depression among Chinese pregnant women with obstetric complications. Psychiatry Res. 2015;226(1):113-9. PMID: 25677395 DOI: 10.1016/j.psychres.2014.12.016

20. Loudon H, Nentin F, Silverman ME. Using clinical decision support as a means of implementing a universal postpartum depression screening program. Arch Womens Ment Health. 2016;19(3):501-5. PMID: 26669601 DOI: 10.1007/s00737015-0596-y

21. Dolatian M, Maziar P, Alavimajd H, Yazdjerdi M. The relationship between mode of delivery and postpartum depression. J Reprod Infertil. 2006;7(3):260-9. [Persian]

22. Bahrami NO, Araban M, Bahrami SO. The impact of antenatal education on postpartum depression, Dezful, Iran. Bim J Hormozgan Univ Med Sci. 2010;13(4):277-83. [Persian]

23. Shayeghian Z, Tabatabaey S. Effect of maternal anxiety during third trimester on pregnancy outcomes and infants' mental health. Hayat. 2008;14(4):57-65. [Persian]

24. Varma D, Chandra PS, Thomas T, Carey MP. Intimate partner violence and sexual coercion among pregnant women in India: relationship with depression and post-traumatic stress disorder. J Affect Disord. 2007;102(1):227-35. PMID: 17109969 DOI: 10.1016/j.jad.2006.09.026

25. Taiwo OJ, Olayinka OO. The validation of edinburgh postpartum depression scale (EPDS) in North Central Nigeria. J Med Tropics. 2007;9(2):29-40. DOI: 10.4314/ jmt.v9i2.35209

26. Montazeri A, Torkan B, Omidvari S. The Edinburgh Postnatal Depression Scale (EPDS): translation and validation study of the Iranian version. BMC Psychiatry. 2007;7(1):11. PMID: 17408479 DOI: 10.1186/1471-244X-7-11

27. Mazhari S, Nakhaee N. Validation of the Edinburgh postnatal depression scale in an Iranian sample. Arch Womens Ment Health. 2007;10(6):293-7. PMID: 18058062 DOI: 10.1007/s00737-007-0204-x

28. Jafarian T, Fathi M, Arshi M, Ghaderi RA. The effect of men's emotionalintelligence on violence against women among married couples. Knowl Res Appl Psychol. 2015;16(4):76-83. [Persian]

29. Latifian M, Arshi M, Alipour F, Ghaedamini Harouni G. Study on relationship of domestic violence with emotional divorce among married females in Tehran. J Soc Work. 2016;5(2):5-12. [Persian]

30. Bacchus L, Mezey G, Bewley S. Domestic violence: prevalence in pregnant women and associations with physical and psychological health. Eur J Obstet Gynecol Reprod Biol. 2004;113(1):6-11. PMID: 15036702 DOI: 10.1016/S03012115(03)00326-9

31. Bonomi AE, Thompson RS, Anderson M, Reid RJ, Carrell D, Dimer JA, et al. Intimate partner violence and women's physical, mental, and social functioning. Am J Prev Med. 2006;30(6):458-66. PMID: 16704938 DOI: 10.1016/j. amepre.2006.01.015

32. Ludermir AB, Valongueiro S, Araújo TVBd. Common mental disorders and intimate partner violence in pregnancy. Rev Saude Publica. 2014;48(1):29-35. PMID: 24789634

33. Ramezani S, Keramat A, Motaghi Z, Khosravi A. The relationship of sexual satisfaction and marital satisfaction with domestic violence against pregnant women. Int $J$ Pediatr. 2015;3(5.2):951-8. DOI: 10.22038/IJP.2015.4858

34. Wasfy A, Al Faisal W, El Sawaf E, Al Serkal F. Intimate 
partner violence against women in: prevalence, associated factors and health consequences, 2012-2013. Midd East $J$ Psych Alzheimers. 2014;5(3):19-27. DOI: 10.5742/MEJPA. 2014.92527

35. Maghsodi S, Yarinasab F, Ebrahimi F. Investigating factors influencing domestic violence against women (case study: city of Kerman). J Soc Dev. 2015;9(3):53-78. [Persian]

36. Moore AR. Types of violence against women and factors influencing intimate partner violence in Togo (West Africa). J Fam Violence. 2008;23(8):777. DOI: https://doi.org/10. 1007/s10896-008-9203-6

37. Khadivzadeh T, Erfanian F. Comparison of domestic violence during pregnancy with the Pre-pregnancy period and its relating factors. Iran $J$ Obstet Gynecol Infertil. 2011;14(4):47-56. [Persian]

38. Koski AD, Stephenson R, Koenig MR. Physical violence by partner during pregnancy and use of prenatal care in rural India. J Health Popul Nutr. 2011;29(3):245. PMID: $\underline{21766560}$ 